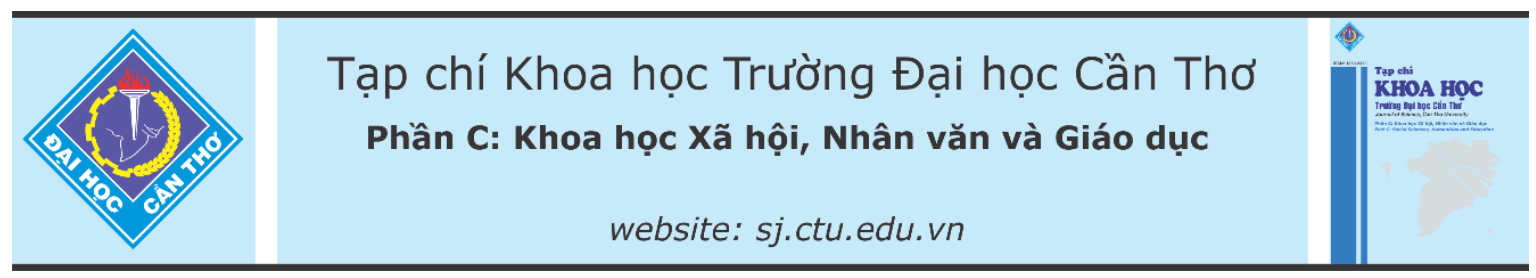

DOI:10.22144/ctu.jvn.2021.024

\title{
TỔNG QUAN NGHIÊN CỨU VỀ KIẾN THỨC ÚNG DỤNG CÔNG NGHỆ THÔNG TIN (TPACK) TRONG DẠY HỌC NGOẠI NGŨ்
}

\author{
Nguyễn Văn Lợi* \\ Khoa Ngoại ngưu, Truờng Đại học Cần Tho \\ *Người chịu trách nhiệm về bài viết: Nguyễn Văn Lọi (email: loinguyen@ctu.edu.vn)
}

\section{Thông tin chung:}

Ngày nhận bài: 26/07/2020

Ngày nhận bài sủa: 15/09/2020

Ngày duyệt đăng: 27/02/2021

\section{Title:}

Technological pedagogical and content knowledge in foreign language teaching: A literature review

\section{Tù khóa:}

Công nghệ thông tin, đào tạo giáo viên, kiến thức, năng lục, ngoại ngũ

\section{Keywords:}

Foreign language, knowledge, teacher education and training, TPACK

\begin{abstract}
This paper is aimed at providing a review of studies related to technological pedagogical content knowledge (TPACK). The literature is based on credible sources especially journal articles published within 20 recent years on reliable journals and publishers. The TPACK model, its roles, and findings of related empirical studies are reviewed and analysed. On that basis, the author stressed the necessity to conduct research on TPACK in the context of Vietnam. Three directions for future research include validating recent TPACK assessment tools, researching foreign language teachers' TPACK, and focusing on TPACK development and its effect on teaching and learning of foreign languages.
\end{abstract}

\section{TÓM TÁT}

Bài viết này phân tích tổng quan về kiến thức ứng dụng công nghệ thông tin (technological pedagogical content knowledge - TPACK) trong day học ngoại ngũ. Dựa trên các nghiên cưu được xuất bản trên các tạp chí quốc tế có uy tín trên thế giới trong 20 năm gần đây, bài viết này thảo luận mô hình TPACK, vai trò của nó cũng nhu nhũng thành tưu đạt được tù nhũng nghiên cứu. Với nhũng hiểu biết tù̀ tổng quan, bài viết đề xuất ba huớng nghiên cứu về TPACK nhằm góp phần nâng cao hiểu biết về lý luận và thực tiễn liên quan đến công tác đào tạo và phát triển chuyên môn cho giáo viên về kiến thức ứng dụng CNTT trong dạy học ngoại ngũ. Ba hướng nghiên cứu bao gồm: xác trị công cu khảo sát TPACK, nghiên cứu TPACK của giáo viên ngoại ngũ và nghiên cứu tác động của đào tạo và tập huấn TPACK đối với dạy và học ngoại ngũ.

\section{GIỚI THIỆU}

Thế kỷ XX đã chứng kiến sự bùng nổ của công nghệ, đặc biệt là công nghệ thông tin và truyền thông. Với sự ra đời của máy tính vào thập niên 50 và sự phát triển mạnh mẽ của nó vào thập niên 80 và 90, nhiều công nghệ ứng dụng máy tính cũng đồng thời xuất hiện phục vụ cho công tác thông tin truyền thông đặc biệt là sự phát triển mạnh mẽ của mạng toàn cầu. Bước sang thế kỷ XXI, cuộc cách mạng công nghệ 4.0 với sự phát triển công nghệ thông tin và truyền thông, công nghệ đám mây đã tác động đến mọi lĩnh vực đời sống, xã hội. Ngoài những kỹ năng, kiến thức chuyên môn, công dân ở thế kỷ XXI cần phải được trang bị những kỹ năng $4 \mathrm{C}$ trong đó kỹ năng sử dụng công nghệ thông tin (CNTT) (UNESCO, 2015). Sự phát triển này đặt ra yêu cầu đổi mới trong công tác giáo dục-đào tạo, đặc biệt là đào tạo giáo viên, góp phần nâng cao nguồn nhân lực cho xã hội. Để đáp ứng những yêu cầu phát triển 
của một xã hội hiện đại, công tác đào tạo và phát triển chuyên môn giáo viên nói chung cũng cần phải cập nhật và đổi mới về những yêu cầu mang tính thời đại. Trong đó việc đổi mới phương thức và hình thức dạy học là một trong những yêu cầu cần thiết.

Trong bối cảnh đó, việc phát triển năng lực ứng dụng CNTT cho giáo viên trong dạy học trở thành một trong những vấn đề được nhiều nhà nghiên cứu giáo dục trên thế giới quan tâm. Hầu hết những thảo luận, nghiên cứu học thuật, nghiên cứu thực tiễn lớp học, đều nhắm đến mục tiêu khai thác các công nghệ thông tin và truyền thông nhằm xúc tiến quá trình dạy học một cách hiệu quả, và giáo viên cần được trang bị những kỹ năng kiến thức gì để có thể vận dụng công nghệ một cách hiệu quả trong dạy học bộ môn. Riêng đối với môn ngoại ngữ, trong vòng 20 năm gần đây, những nghiên cứu về năng lực ứng dụng CNTT trong dạy học của giáo viên đã mang lại một số hiểu biết quan trọng. Bài viết này nhằm cung cấp một cái nhìn tổng quan về những thành tựu nghiên cứu liên quan đến khả năng ứng dụng CNTT trong dạy học tiếng Anh của giáo viên trên thế giới. Qua đó bài viết sẽ rút ra một số kết luận và đề xuất liên quan đến nghiên cứu và đào tạo giáo viên về ứng dụng CNTT trong dạy học ngoại ngữ tại Việt Nam. Tổng quan tài liệu được dựa trên các nghiên cứu xuất bản trên các tạp chí, sách của nhà xuất bản có uy tín, cơ sở dữ liệu của nhà xuất bản Routledge, Sage và Elsevier trong vòng 20 năm kể từ khi Mishra và Koehler (2006) xuất bản ấn phẩm về mô hình TPACK. Do giới hạn của bài viết, các nghiên cứu được chọn sử dụng cho tổng quan thuộc lĩnh vực dạy ngoại ngữ, đặc biệt là tiếng Anh (EFL). Bài viết sẽ gồm các phần sau: mô hình kiến thức ứng dụng CNTT, tổng quan nghiên cứu về kiến thức ứng dụng CNTT của giáo viên trong lĩnh vực dạy học ngoại ngữ.

\section{MÔ HÌNH KIẾN THỨC ÚNG DỤNG CNTT TRONG DẠY HỌC}

Phần này bao gồm khái niệm kiến thức ứng dụng CNTT trong dạy học, vai trò của mô hình kiến thức ứng dụng CNTT trong dạy học; và phương pháp đánh giá kiến thức ứng dụng CNTT trong dạy học.

\subsection{Kiến thức ứng dụng CNTT trong dạy học (TPACK)}

Định nghĩa khái niệm kiến thức ứng dụng CNTT trong dạy học- TPACK (Technological pedagogical content knowledge) không thể không đề cập đến khái niệm 'năng lực' (tiếng Anh là competence/ competency). Theo Bộ giáo dục-đào tạo Úc (Department of Education and Training, n.d), năng lực là sự ứng dụng kiến thức và kỹ năng có tính ổn định nhất quán theo một chuẩn thực hành qui định. Theo Khung năng lực của UNESCO (2015), và Khung năng lực toàn cầu của tổ chức hợp tác và phát triển kinh tế OECD (2018), năng lực là sự liên kết giữa kiến thức, kỹ năng và thái độ và những giá trị, dẫn đến những hành vi không thể thiếu để thực thi một công việc. Hoàng Huy Bình (2015) kết luận rằng năng lực biểu hiện qua hành động và hiệu quả thực hiện hành động. Nguyễn Phương Lan trích trong Hoàng Huy Bình (2015), cho rằng kiến thức, kỹ năng và thái độ là cấu trúc bề mặt của năng lực, và việc huy động kiến thức kỹ năng thái độ để thực thi một công việc hiệu quả là cấu trúc tiềm ẩn của năng lực. Theo Đặng Thành Hưng (2012, trích trong Hoàng Huy Bình, 2015), kiến thức thể hiện năng lực hiểu, kỹ năng thể hiện năng lực làm, và thái độ thể hiện năng lực cảm xúc. Như vậy về cấu trúc, có thể thấy kiến thức là một thành tố hình thành nên năng lực.

Theo phiên bản chỉnh sửa về thang nhận thức của Bloom (Anderson and Krasthwohl, 2001), quá trình nhận thức hay tiếp thu kiến thức được mô tả theo từng thang bậc hay trình độ: từ ghi nhớ hay biết (knowledge), hiểu, ứng dụng, cho đến như phân tích, tổng hợp, phê phán và sáng tạo. Theo đó, mỗi thang bậc có thể hiểu là một bậc năng lực nhận thức, trong đó ghi nhớ là một bậc năng lực nhận thức thấp nhất, và sáng tạo là bậc năng lực nhận thức cao nhất. Biết là tiền đề cho các bậc nhận thức theo sau được gọi kỹ năng (skills) và khả năng (abilities) (Armstrong, 2010). Kỹ năng và khả năng này khác với kỹ năng thao tác vận động trong thang kỹ năng (psychomotor domain) của Bloom. Theo Anderson and Krasthwohl (2001), kiến thức có thể được phân chia thành kiến thức thông tin (factual knowledge), kiến thức khái niệm (conceptual knowledge), kiến thức qui trình (procedural knowledge) và kiến thức về quá trình nhận thức (metacognitive knowledge). Như vậy, thuật ngữ kiến thức/sự hiểu biết (knowledge) có thể hiểu là khả năng biết về $\mathrm{X}$, làm tiền đề cho những quá trình nhận thức $\mathrm{Y}, \mathrm{Z}$.

Trong bài viết này thuật ngữ kiến thức (knowledge), viết tắt là $\mathrm{K}$ trong mô hình TPACK không chỉ có nghĩa là nhớ hay biết, mà bao hàm cả khả năng ứng dụng, triển khai các hoạt động giảng dạy có ứng dụng CNTT. Mô hình này, tạm dịch là kiến thức/năng lực ứng dụng CNTT trong dạy học, đã được nhiều học giả trong nhiều lĩnh vực đào tạo giáo viên trên thế giới quan tâm nghiên cứu và phát triển (Abbitt, 2011; Harris \& Hofer, 2011; 
Rosenberg \& Koehler, 2015; Saltan \& Arslan, 2017; Voogt \& McKenney, 2017).

TPACK là chữ viết tắt của từ 'Total PACKage' được Thompson và Mishra (2009, trích trong Tai, 2015) sử dụng để chỉ năng lực ứng dụng CNTT như là một 'gói' kiến thức tích hợp. Từ gốc của nó là TPCK (technological pedagogical content knowledge). Sự ra đời của TPACK dựa trên ý tưởng của Shulman (1987) về kiến thức sư phạm bộ môn (pedagogical content knowledge- PCK) được tích hợp từ kiến thức môn học (content knowledge- $\mathrm{CK}$ ) và kiến thức sư phạm (pedagogical knowledge- $\mathrm{PK}$ ). Theo đó, mỗi một môn học đều có những đặc thù riêng, và người giáo viên cần phải được trang bị kiến thức hay năng lực vận dụng các phương pháp, biện pháp, và kỹ thuật dạy học phù hợp với đặc thù chuyên môn để chuyển tải nội dung chuyên môn một cách hiệu quả. Trong dạy học ngoại ngữ, PCK là kiến thức về phương pháp dạy học ngoại ngữ gắn kết với hiểu biết về bản chất của ngôn ngữ, quá trình tiếp thu ngôn ngữ và tác động của bối cảnh trong việc dạy và học ngoại ngữ.

Cùng với ý tưởng đó và sự quan sát sau 5 năm nghiên cứu về việc đào tạo kiến thức công nghệ một cách biệt lập, không gắn kết với kiến thức sư phạm môn học, Mishra and Koehler (2006) đã đề xuất mô hình TPACK mô tả sự gắn kết giữa kiến thức công nghệ với kiến thức sư phạm môn học (Hình 1). Về cơ bản, mô hình TPACK dựa trên sự kết hợp giữa 3 lĩnh vực kiến thức tổng hợp bao gồm: kiến thức sư phạm môn học (pedagogical content knowledgePCK), kiến thức công nghệ môn học (technological content knowledge- TCK), kiến thức sư phạm về công nghệ (technological pedagogical knowledge$\mathrm{TPK}$ ); ba thành phần kiến thức tích hợp này là sự đan xen kết hợp giữa ba loại kiến thức nền tảng là $\mathrm{CK}, \mathrm{PK}$ và $\mathrm{TK}$ (technological knowledge -kiến thức công nghệ).

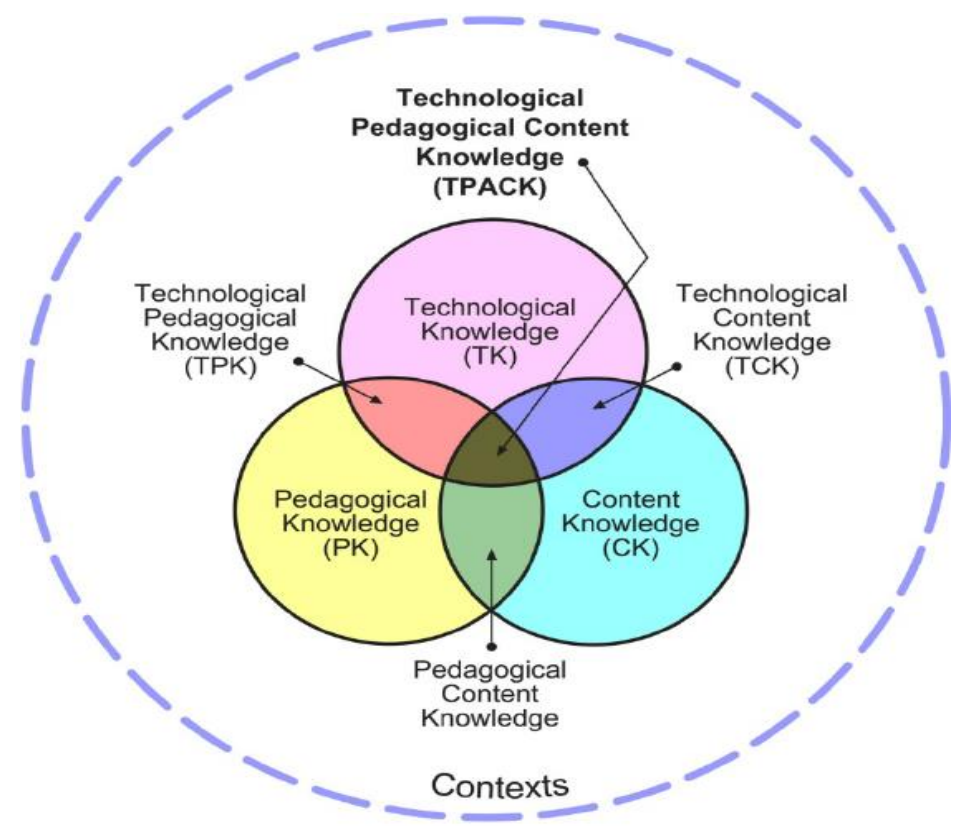

Hình 1. Mô hình TPACK (Mishra and Koehler, 2006, reproduced by permission of the publisher, (C) 2012 by tpack.org)

Như đã nói ở trên PCK là năng lực hiểu và vận dụng các phương pháp dạy học đặc thù của môn học nhằm truyền đạt nội dung môn học một cách hiệu quả. TCK là sự hiểu biết về các công nghệ có thể ứng dụng trong dạy học một môn học chuyên biệt. Nó khác biệt với kiến thức về công nghệ nói chung bao gồm những hiểu biết cơ bản về phần cứng và phần mềm máy tính, lưu trữ thông tin, hay vận hành các máy chụp ảnh quay phim, v.v., Không phải tất cả các công nghệ đều phù hợp để dạy học tất cả các môn học. Ví dụ trong dạy học ngoại ngữ, các công nghệ nào hỗ trợ quá trình nhận thức ngôn ngữ như tạo hứng thú, gây chú ý, thúc đẩy tương tác sẽ là những công nghệ có thể khai thác. TPK là kiến thức về vai trò và tiềm năng sư phạm của các loại công nghệ trong dạy học ngoại ngữ, về cách khai thác các công nghệ phù hợp để tác động đến quá trình nhận thức của học sinh. Ví dụ đối với dạy học ngoại ngữ, ứng dụng web Wiki hỗ trợ tốt cho sự tương tác và hợp tác, một quá trình học tập phù hợp với lý thuyết 
tiếp nhận ngôn ngữ thông qua tương tác xã hội (Long, 1996). Khả năng vận dụng kết hợp giữa 3 loại kiến thức tổng hợp trên sẽ tạo nên cái gọi là kiến thức ứng dụng CNTT trong dạy học bộ môn (TPACK). Như vậy, TPACK là một loại kiến thức/năng lực kết hợp từ 6 lĩnh vực kiến thức: $\mathrm{CK}$, TK, PK, TCK, TPK, PCK. Loại kiến thức này chịu sự chi phối của sự hiểu biết và tương tác với bối cảnh dạy học cụ thể (context). Tùy vào trình độ, cấp độ và bối cảnh thực tế dạy học, việc ứng dụng CNTT cần được điều chỉnh cho phù hợp.

\subsection{Vai trò của TPACK}

Nghiên cứu về TPACK đem lại một số lợi ích nhất định. Theo một số nhà nghiên cứu (Koehler \& Mishra, 2008; Niess, 2008; Shih \& Chuang, 2013) được trích trong Tseng (2015), cần phải có một mô hình và công cụ đo lường TPACK đã được thẩm định về tính giá trị và độ tin cậy vì điều này giúp giáo viên tự đánh giá việc sử dụng CNTT trong dạy học nhằm nâng cao chất lượng giảng dạy. Ngoài ra, tìm hiểu về TPACK của giáo viên giúp các nhà nghiên cứu và đào tạo hiểu rõ khả năng vận dụng CNTT trong dạy học của giáo viên, qua đó xây dựng chương trình tập huấn và phát triển chuyên môn. Nghiên cứu của Hsu (2016) tìm hiểu mối liên hệ giữa kiến thức TPACK và việc sử dụng thiết bị di động trong dạy học tiếng Anh của 158 giáo viên tiếng Anh tại Đài Loan tham gia khảo sát về TPACK và mô hình tiếp nhận công nghệ trong dạy học TAM (Technology Acceptance Model). Kết quả cho thấy kiến thức TPACK của giáo viên ảnh hưởng đến nhận thức của họ về sự hữu dụng và tiện lợi của thiết bi di động trong dạy học ngoại ngữ. Mối liên hệ này ảnh hưởng đến khả năng duy trì việc thực hiện dạy học thông qua thiết bị di động. Nghiên cứu trong lĩnh vực khác cũng khẳng định thêm vị trí của TPACK trong đào tạo và phát triển chuyên môn cho giáo viên (Harris \& Hofer, 2011).

\subsection{Nhận xét về mô hình TPACK}

Mô hình TPACK đã cho thấy quan điểm tiếp cận về việc sử dụng CNTT trong dạy học theo hướng cụ thể hóa vai trò của từng loại kiến thức. Dựa trên mô hình, các chuẩn kiến thức có thể được xây dựng giúp cho việc đánh giá và tự đánh giá trong đào tạo và phát triển chuyên môn. Ngoài ra, mô hình TPACK đã cho thấy quan điểm ứng dụng CNTT trong dạy học theo hướng tích hợp và biến đổi. Thay vì đào tạo kiến thức về công nghệ riêng biệt, các chương trình đào tạo và phát triển chuyên môn cho giáo viên cần lưu ý về sự gắn kết giữa kiến thức sư phạm môn học và kiến thức CNTT nhằm khai thác tính năng hỗ trợ và sự tương thích của công nghệ đối với quá trình và phương pháp day-học một môn học hay một nội dung kiến thức, kỹ năng cụ thể (Mishra \& Koehler, 2006). Nguyên lý dạy và học bộ môn không thể tách rời với việc ứng dụng CNTT (Levy, 1997), kiến thức và kỹ năng về CNTT đơn thuần không thể giúp người giáo viên ứng dụng CNTT một cách hiệu quả.

Trong lĩnh vực dạy học ngoại ngữ với sự hỗ trợ của máy tính (Computer Assisted Language Learning-CALL), quan điểm kết hợp công nghệ với phương pháp sư phạm đã được nhấn mạnh từ lâu (xem Levy, 1997; Chapelle, 2003; Davies, 2012). Levy (1997) đề xuất sự tương thích (alignment) giữa việc khai thác và ứng dụng $\mathrm{CNTT}$ với mục tiêu giảng dạy, nội dung kiến thức và phương pháp dạy học ngoại ngữ. Chapelle (2003) đề xuất sự gắn kêt giữa việc ứng dụng CNTT với kiến thức về quá trình dạy và học, và kiến thức phát triển tài liệu giảng dạy. Theo đó, quá trình học tập ngoại ngữ bao gồm quá trình nhận thức (chú ý, hiểu, phát hiện lỗ hỏng trong kiến thức của bản thân) và quá trình tương tác xã hội (tương tác với người học, giáo viên và tài liệu học tập) cần phải được thúc đẩy bằng cách khai thác các ứng dụng CNTT một cách hiệu quả, gắn kết với tiến trình dạy học (trình bày, tương tác, phản hồi/đánh giá). Tương tự, Davies (2012) và cộng sự thông qua dự án ICT4LT project phát triển một trang web (www.ict4lt.com) nhằm hỗ trợ giáo viên ngoại ngữ trên thế giới về ứng dụng CNTT, cũng nhấn mạnh rằng việc chọn lựa công cụ dạy học trong dự án nhằm thúc đẩy hiệu quả dạy học của các phương pháp dạy ngoại ngữ. Để làm được điều này, người giáo viên cần phải biết vận dụng CNTT để thiết kế hoạt động dạy học (activity/task) sao cho có thể kích hoạt được các quá trình nhận thức và xã hội đã nêu.

Tuy nhiên, mô hình này không phải không có những ý kiến phê bình. Theo Willermark (2018), mô hình TPACK chưa có tính thực tiễn cao vì khái niệm này chưa được hiểu và mô tả đầy đủ. Một số nghiên cứu đã chỉ ra ranh giới giữa các lĩnh vực kiến thức trong mô hình chưa được xác định rõ ràng (xem Tseng, 2015). Theo Parr et al (2013), TPACK vừa có vẻ manh mún, chia cắt và phân loại các loại kiến thức, vừa đồng thời gắn kết chúng lại, và nó dường như khuyến khích một quan điểm 'cứng nhắc' về kiến thức chuyên môn, với giả định rằng giáo viên là người tiếp nhận thụ động, không có vai trò gì trong việc phát triển và triển khai kiến thức môn học. Quan điểm này xem TPACK như là một năng lực kiến tạo mà mỗi cá nhân giáo viên phát triển trong bối cảnh ứng dụng thực tiễn (xem Harris et al., 2017). 
Các nghiên cứu khác nhau về TPACK nhằm phát triển các mô hình riêng biệt cho từng lĩnh vực như TPACK-geography, TPACK-EFL, TPACK-web thể hiện một sự thụt lùi trong việc thống nhất một lý thuyết chung (Harris et al., 2017; Schmid et al., 2020). Tuy nhiên, theo quan điểm của người viết, việc phát triển các mô hình riêng biệt trong từng lĩnh vực sẽ có thuận lợi hơn trong việc đảm bảo tính giá trị và xác thực của mô hình. Vì vậy, cần có nhiều nghiên cứu nhằm phát triển mô hình $\mathrm{TPACK}$ trong dạy học ngoại ngữ đặc biệt là bối cảnh của Việt Nam.

2.4. Phương pháp nghiên cứu và công cụ đánh giá năng lực TPACK

\subsubsection{Phương pháp và công cu nghiên cứu}

Nhiều phương pháp nghiên cứu đã được thực hiện sử dụng các công cụ đo lường TPACK khác nhau. Vì vậy, dựa trên những kết quả khoa học đã được tổng hợp giúp định hướng rõ nét hơn phương pháp đo lường TPACK. Theo hướng này, có hai nghiên cứu tổng hợp các phương pháp nghiên cứu được tìm thấy. Nghiên cứu tổng quan của Willermark (2018) được sử dụng để củng cố lập luận và nghiên cứu của Koehler, Shin and Mishra (2012) làm nền tảng tổng quan vì các lý do sau đây:

- Đây là một nghiên cứu phân tích tổng hợp (meta-analysis) có mô tả phương pháp thực hiện, và phân tích một cách cụ thể và khoa học.

- Nghiên cứu này tổng hợp 66 nghiên cứu chọn lọc từ trên 300 nghiên cứu từ nhiều nguồn khác nhau xuất bản từ 2006 đến 2010 , kể từ thời điểm mô hình TPACK được xuất bản. Các nghiên cứu được chọn lọc là các nghiên cứu trực tiếp về TPACK trong nhiều lĩnh vực chuyên môn khác nhau.

Các phương pháp đều được các tác giả xem xét về hai khía cạnh: độ tin cậy và tính giá trị/chuẩn xác. Kết quả phân tích của các tác giả trên cho thấy các phương pháp và công cụ đánh giá TPACK phổ biến bao gồm: khảo sát tự đánh giá, khảo sát mở, đánh giá thực hành, phỏng vấn và quan sát. Koehler et al. (2012) cũng lưu ý rằng bốn phương pháp như khảo sát tự đánh giá, phỏng vấn, đánh giá thực hành và quan sát được sử dụng nhiều hơn khảo sát mở.

Đối với phương pháp khảo sát mở (open-ended questionnaire) trong đó các câu hỏi mở về kiến thức CNTT ví dụ như 'đâu là điểm mạnh và điểm hạn chế của...?' được sử dụng, thì chỉ có 3 trên 20 nghiên cứu thể hiện độ tin cậy thông qua kỹ thuật mã hóa dự liệu chéo (interrater reliability) và chỉ có một nghiên cứu giải quyết vấn đề tính giá trị của công cụ đo lường TPACK. Theo các tác giả thì khảo sát mở gây khó khăn cho việc phân tích số liệu, nên điều này ảnh hưởng đến tính giá trị.

Mặc dù nhấn mạnh rằng tiềm ẩn bên dưới nhiều nghiên cứu là giả định sử dụng kết hợp phương pháp (triangulation), các tác giả cho rằng giả định này cần được làm rõ thông qua minh chứng cụ thể về TPACK thể hiện qua dữ liệu của từng phương pháp. Qua phân tích, các tác giả kết luận rằng phần lớn các nghiên cứu không đưa ra được minh chứng (evidence) về độ tin cậy và tính giá trị.

Phương pháp quan sát giúp tìm hiểu thực tiễn về năng lực thực hành ứng dụng CNTT của giáo viên và sự phát triển của năng lực này qua thời gian (Koehler et al., 2012), nhưng hạn chế của phương pháp quan sát là sự khó tiếp cận và sự thay đổi hành vi của người tham dự, đặc biệt là trong bối cảnh dạy học ở Việt Nam. Cũng vậy, phương pháp đánh giá thực hành thông qua phân tích giáo án, công nghệ sử dụng cũng khó có thể thực hiện được.

Khảo sát đóng là một trong những phương pháp phổ biến. Nghiên cứu tổng quan của Willermark (2018) cũng khẳng định rằng phương pháp khảo sát tự đánh giá (self-reporting) được sử dụng phổ biến trong nghiên cứu đo lường TPACK, trong khi đánh giá TPACK qua các hoạt động dạy học hầu như không được sử dụng. Theo Koehler et al. (2012), đa số các nghiên cứu (11/12) sử dụng công cụ tự đánh giá đo lường độ tin cậy bằng chỉ số Cronbach's Alpha và cho kết quả giá trị cao từ .92 đến .99 ; đa số các nghiên cứu kiểm định tính giá trị của công cụ thông qua phân tích nhân tố khám phá và khẳng định.

Phỏng vấn sâu giúp xác định chiều sâu về kiến thức TPACK của giáo viên. Vì thế một nghiên cứu đáng tin cậy về TPACK cần kết hợp sử dụng các phương pháp khác nhau, trong đó phương pháp khảo sát tự đánh giá và phỏng vấn để xác định TPACK của giáo viên là một sự kết hợp đáng tin cậy.

\subsubsection{Thang đánh giá thông qua khảo sát}

Đa số các công cụ tự đánh giá hay khảo sát sử dụng một thang đo giống nhau: Thang điểm 5 chỉ mức độ đồng ý $(1=$ hoàn toàn không đồng ý, $5=$ hoàn toàn đồng ý) đề khảo sát mức độ đồng ý đối với sự hiểu biết về TPACK được mô tả bằng những từ ngữ như 'tôi biết về...', hay 'tôi có thể...'. Tuy nhiên thang đo này khá mơ hồ trong việc xác định mức hiểu biết, vì mục đích của nó là nhằm xác trị (validate) công cụ thông quan phân tích nhân tố. Hạn chế của nó là câu trả lời 'đồng ý' hay 'không đồng ý' không cung cấp thông tin rõ ràng về mức độ hiểu biết của giáo viên đối với một khía cạnh kiến thức hay kỹ năng cụ thể. Thí dụ đối với câu hỏi 'Tôi 
có thể sử dụng các phần mềm văn phòng', câu trả là 'đồng ý' cũng chỉ có thể xác định là người trả lời có thể sử dụng; nhưng câu trả lời không thể cho ta biết chính xác về khả năng sử dụng phần mềm của người trả lời. Đối với việc tìm hiểu vể TPACK của giáo viên nhằm phục vụ tốt cho công tác đào tạo trong một bối cảnh cụ thể, cần phải điều chỉnh thang đo theo các mức năng lực. Như đã nói ở trên, năng lực có thể được mô tả theo thang bậc. Dựa trên cơ sở phân cấp tương tự với thang Bloom, một thang đo mức độ kiến thức về các CNTT có thể được hình thành và thử nghiệm. Ví dụ đối với kiến thức công nghệ cơ bản (TK) có thể gồm:

1. 'Tôi chưa từng nghe về...' để chỉ mức độ biết thấp nhất, có thể hiểu là mức không biết/không có kiến thức, hay mức độ năng lực rất yếu.

2.'Tôi biết nhưng chưa từng sử dụng' thể hiện mức năng lực có biết nhưng không xác định được năng lực sử dụng.

3. 'Tôi có thể sử dụng, nhưng cần phải nổ lực' có thể hiểu là một mức độ năng lực trung bình.

4. 'Tôi có thể sử dụng, nhưng không hướng dẫn người khác được' tương đương khá thành thạo; và

5. 'Tôi có thể sử dụng và hướng dẫn người khác' được hiểu là mức độ thành thạo về năng lực cao nhất.

\section{TỔNG QUAN VỀ CÁC NGHIÊN CÚU LIÊN QUAN ĐẾN TPACK TRONG DẠY HỌC NGOẠI NGŨ'}

Nhiều nghiên cứu đã tìm hiểu TPACK trong nhiều bối cảnh bao gồm các đối tượng tham gia như giáo viên và sinh viên sư phạm thuộc các chuyên môn khác nhau. Tuy nhiên, đối với môn ngoại ngữ, đặc biệt là tiếng Anh, chỉ có một vài nghiên cứu xuất bản trong 10 năm gần đây. Có thể phân các nghiên cứu thành hai cụm: (1) Các nghiên cứu về xác trị khái niệm và công cụ đánh giá TPACK trong dạy học ngoại ngữ (cụ thể là tiếng Anh) (2) Các nghiên cứu về nhận thức hay đánh giá của giáo viên hoặc giáo sinh về TPACK.

\subsection{Nghiên cứu xác trị khái niệm và công cụ đánh giá TPACK}

Xác trị khái niệm và công cụ đánh giá TPACK đã được thực hiện trong lĩnh vực đào tạo giáo viên nói chung. Nghiên cứu của Schmidt et al. (2009) về phát triển công cụ khảo sát TPACK không mang tính đặc thù của môn học, và là công cụ điển hình được nhiều nghiên cứu trong lĩnh vực dạy học ngoại ngữ điều chỉnh sử dụng. Công cụ khảo sát TPACK gồm 75 câu hỏi và trải qua quá trình thẩm định và xác trị bằng phân tích nhân tố, 18 câu đã bị loại. Khách thể khảo sát trong nghiên cứu này là sinh viên sư phạm ngành tiểu học và mầm non tham gia vào một khóa học sử dụng CNTT trong dạy học. Tuy nhiên, theo Bostancioğlu và Handley (2018), các nghiên cứu phát triển công cụ đánh giá TPACK dựa trên bảng khảo sát của Schmidt et al. (2009) đều không thành công trong việc rút trích 7 nhân tố tìm ẩn dưới TPACK, trong đó $\mathrm{PK}$ là nhân tố chưa được phân định rõ ràng nhất.

Có hai nghiên cứu xác trị thông qua sự đánh giá của học sinh đối với TPACK của giáo viên trong dạy học tiếng Anh. Tseng (2016) thiết kế công cụ khảo sát dựa trên tổng quan tài liệu nhằm đo lường TPACK của giáo viên tiếng Anh thông qua sự đánh giá của 57 học sinh trung học đối với 3 giáo viên tiếng Anh của học tại Đài Loan. Khảo sát gồm 35 câu hỏi yêu cầu học sinh trả lời theo thang điểm 5 (1 hoàn toàn không đồng ý, 5 hoàn toàn đồng ý). Bảng hỏi được dịch sang tiếng mẹ đẻ, thẩm định bởi các chuyên gia, trước khi được tính toán bằng phân tích thống kê. Kết quả cho thấy mặc dù công cụ có chỉ số Cronbach alpha cao, phân tích nhân tố khám phá chỉ cho thấy có 5 nhân tố xuất hiện trong tổng số 7 nhân tố thuộc 7 lĩnh vực kiến thức TPACK. Đây là điểm hạn chế của nghiên cứu như tác giả đã công nhận. Nguyên nhân là học sinh có thể không phân biệt được các khái niệm về kiến thức liên quan đến TPACK. Kết quả này khác với nghiên cứu của Shih and Chuang (2013) đối với sinh viên cao đẳng cho thấy các lĩnh vực kiến thức được phân biệt một cách rõ ràng (construct validity).

Đối với khách thể là giáo sinh/sinh viên sư phạm tiếng Anh, Baser et al. (2016) nghiên cứu xác trị công cụ khảo sát TPACK gồm 50 câu hỏi thông qua 2 vòng: vòng 1 với sự tham gia của 174 sinh viên sư phạm tiếng Anh năm 3 và 4 , vòng 2 là 204 giáo sinh năm 3 và 4 . Trước khi khảo sát, công cư được thẩm định về nội dung bởi các chuyên gia. Bối cảnh khảo sát là một trường đại học ở Thổ Nhĩ Kỳ. Kết quả rút trích thông qua phân tích nhân tố khám phá cho thấy có 39 câu tạo thành 5 nhân tố. Phân tích nhân tố khẳng định vòng 2 sau khi loại bỏ các câu không đạt giá trị thống kê, cho kết quả 7 nhân tố trong mô hình TPACK.

Đối với khách thể là giáo viên, Chai et al. (2013) là nghiên cứu đầu tiên xác trị công cụ đo lường TPACK trong bối cảnh Singapore, đối với giáo viên tiếng Trung là ngôn ngữ thứ hai, và rút trích thành công 7 nhân tố/thành phần kiến thức trong TPACK. Tuy nhiên, theo Bostancioğlu and Handley (2018), hạn chế của nghiên cứu này là công cụ khảo sát dựa 
trên quan điểm dạy học kiến tạo, trong khi đó công nghệ là để hỗ trợ quá trình dạy học và không phụ thuộc vào một quan điểm duy nhất. Theo quan điểm này, Bostancioğlu and Handley (2018) thiết kế một công cụ khảo sát TPACK không thiên về một quan điểm dạy học ngoại ngữ nào duy nhất. Công cụ khảo sát/tự đánh giá được thiết kế và đánh giá một cách khoa học. Giai đoạn 1 là xây dựng 76 câu hỏi dựa trên tham khảo nhiều nghiên cứu đáng tin cậy, chương trình đào tạo về $\mathrm{CNTT}$, chuẩn CNTT giành cho giáo viên của Mỹ và Úc. Kế đến là sự tham gia thẩm định nội dung (content validation) các câu hỏi khảo sát của 36 chuyên gia trong lĩnh vực ứng dụng CNTT trong dạy học ngoại ngữ (CALL). Từ phản hồi của các chuyên gia và phân tích tỉ lệ phù hợp của nội dung (content value ratio), 11 câu bị loại bỏ, còn lại 65 câu hỏi. Giai đoạn cuối cùng là khảo sát và thẩm định tính giá trị và độ tin cậy (construct validation) của công cụ trên diện rộng, với sự tham gia trả lời phiếu khảo sát của 542 giáo viên tiếng Anh từ 72 quốc gia, trong đó có 144 giáo viên là người bản xứ nói tiếng Anh đang công tác tại các quốc gia nơi tiếng Anh là một ngoại ngữ (EFL). Phân tích nhân tố khám phá được sử dụng đối với một phần dữ liệu và phân tích nhân tố khẳng định được sử dụng đối với phần dữ liệu còn lại, để rút trích các nhân tố tiềm ẩn, đại diện cho các lĩnh vực kiến thức thuộc TPACK. Kết quả cho thấy có 36 câu hỏi nhóm thành 6 nhân tố. Trong đó $\mathrm{PK}$ và $\mathrm{PCK}$ không có sự phân biệt rõ ràng. Mặc dù vậy, nghiên cứu này không nhằm mục đích đo lường $\mathrm{TPACK}$ của giáo viên tiếng Anh. Hơn nữa đối tượng tham gia mặc dù là giáo viên công tác trong bối cảnh dạy tiếng Anh như một ngoại ngữ (EFL), nhưng theo đề xuất của Bostancioğlu and Handley (2018), công cụ này cần được xác trị trên diện rộng hơn về bối cảnh và đối tượng.

\subsection{Nghiên cứu về đánh giá và phát triển kiến thức TPACK của giáo viên ngoại ngữ}

Các nghiên cứu về đánh giá và phát triển TPACK tập trung vào hai đối tượng là giáo $\sinh / \sinh$ viên sư phạm (pre-service) và giáo viên đang tại chức (in-service).

Đối với khách thể là sinh viên/giáo sinh tiếng Anh, nhiều nghiên cứu tại Thổ Nhĩ Kỳ cho thấy kết quả khác nhau. Öz (2015) khảo sát TPACK của 76 sinh viên sư phạm tiếng Anh trong chương trình đào tạo tại Thổ Nhĩ Kỳ, sử dụng công cụ khảo sát của Schmidt et al. (2009) có điều chỉnh gồm 34 câu. Kết quả cho thấy mức độ hiểu biết của sinh viên về TPACK là khá cao $(M>3.5)$, và sinh viên nữ có hiểu biết cao hơn về PK và TK. Saricoban et al. (2019) đo lường TPACK của 77 sinh viên sư phạm tiếng Anh như một ngoại ngữ, sử dụng công cụ khảo sát điều chỉnh từ Baser et al (2016) gồm 39 câu hỏi tập trung vào 7 lĩnh vực KT thuộc TPACK. Kết quả cho thấy sinh viên có kiến thức TPACK ở mức khá cao trong tất cả các lĩnh vực. Khác với nghiên cứu của Öz (2015), sinh viên nam giỏi về kiến thức công nghệ hơn sinh viên nữ. Ngoài ra, nghiên cứu này tìm thấy tuổi tác tỉ lệ thuận với kiến thức công nghệ; sinh viên lớn tuổi có số điểm tự đánh giá cao hơn về TPACK. Nghiên cứu của Kurt et al. (2013) chứng minh rằng chương trình đào tạo ứng dụng $\mathrm{CNTT}$ sử dụng phương pháp thiết kế thực hành đã giúp sinh viên tiếng Anh gia tăng hiểu biết về TK, TCK, TPK and TPACK sau 12 tuần.

Đối với khách thể là giáo viên, nghiên cứu cũng chỉ ra các kết quả khác biệt về TPACK. Kozikoğlu and Babacan (2019) khảo sát 721 giáo viên tiểng Anh tại Thổ Nhĩ Kỳ thấy rằng giáo viên có mức độ hiểu biết cao về TPACK. Ngoài ra kinh nghiệm giảng dạy không tạo ra sự khác biệt về TPACK giữa các giáo viên. Nazari et al. (2019) nghiên cứu sự khác biệt về TPACK giữa 427 giáo viên tiếng Anh có kinh nghiệm và giáo viên ít kinh nghiệm tại Teheran. Hai công cụ sử dụng là phiếu khảo sát dựa trên công cụ của Schmidt et al. (2009) và phỏng vấn sâu. Kết quả cho thấy giáo viên có nhiều kinh nghiệm có điểm số cao hơn về $\mathrm{PK}$ và $\mathrm{PCK}$, nhưng ngược lại nhóm giáo viên ít kinh nghiệm có điểm số cao hơn về TK, TCK, TPK và TPACK. Tuổi tác tỉ lệ nghịch với kiến thức về TPACK. Prasojo et al (2020) khảo sát 573 giáo viên tiếng Anh tại Indonesia sủ dụng công cụ 28 câu hỏi gồm 7 nhân tố. Kết quả cho thấy giáo viên có kiến thức công nghệ dưới trung bình, mặc dù có đủ kiến thức sư phạm. Giới tính và tuổi tác là hai yếu tố tạo nên sự khác biệt vè năng lực TPACK giữa các giáo viên. Giáo viên nữ có năng lực TPACK cao hơn giáo viên Nam, trong khi đó giáo viên trẻ dưới 35 tuổi có năng lực TPACK cao hơn giáo viên lớn tuổi (> 35), một kết quả giống Nazari et al (2019). Nghiên cứu của Tai (2015) tại Đài Loan cho thấy giáo viên tiếng Anh bậc tiểu học phát triển năng lực TPACK sau khi tham gia các hội thảo tập huấn sử dụng mô hình TPACK trong thực hành thiết kế hoạt động dạy học có ứng dụng CNTT.

Trong bối cảnh Việt Nam, nhiều hoạt động tập huấn về sử dụng CNTT trong dạy học ngoại ngữ (chủ yếu là tiếng Anh) đã được triển khai từ năm 2012 thông qua Đề án Ngoại Ngữ Quốc Gia 2020, nhưng đến nay chưa có nghiên cứu tìm hiểu kiến thức/năng lực ứng dụng CNTT của giáo viên đặc biệt là giáo viên tiếng Anh đang công tác tại các 
trường phổ thông hay sự phát triển về năng lực của giáo viên tiếng Anh như thế nào. Nghiên cứu của Le and Song (2018) sử dụng khảo sát, phỏng vấn và phân tích chương trình đào tạo đối với 42 sinh viên sư phạm tiếng Anh tại một trường đại học cho thấy mặc dù nội dung đào tạo khá tương thích với kiến thức TPACK, nhưng kết quả chứng minh kiến thức TPACK của sinh viên không thay đổi sau khi hoàn thành học phần ứng dụng CNTT trong dạy học tiếng Anh. Tuy nhiên, kết quả phỏng vấn sâu cho thấy các yếu tố như chính sách giáo dục, cơ sở vật chất và niềm tin sư phạm của sinh viên ảnh hưởng đến thái độ ứng dụng CNTT của sinh viên trong dạy học.

\section{NHẬN XÉT VÀ KẾT LUẬN}

Mặc dù bài viết này tổng hợp kết quả của nhiều nghiên cứu đại diện, nhưng do hạn chế về tiếp cận nguồn tài liệu tham khảo tại Việt Nam, một số nghiên cứu khác chưa được lược khảo. Tuy nhiên, từ tổng quan trên có thể có mấy kết luận sau đây:

Đa số có nghiên cứu xác trị/kiểm định khái niệm và công cụ khảo sát TPACK đều dựa trên nền tảng tổng hợp tài liệu về khung TPACK và trải qua quá trình thẩm định có tính khoa học. Mặc dù các công cụ khảo sát có số câu hỏi khác nhau, kết quả cho thấy đa số các công cụ đều cho kết quả khá tin cậy. Trong số đó, nghiên cứu gần đây nhất của Bostancioğlu và Handley (2018) có sự tham gia thẩm định của một số lượng lớn các chuyên gia, đồng thời được phân tích thống kê qua hai giai đoạn: phân tích nhân tố khám phá và phân tích nhân tố khẳng định để kiểm định giá trị và độ tin cậy của công cụ (construct validity and scale reliability). Ngoài ra, công cụ này được khảo sát trên đối tượng giáo viên tiếng Anh tại nhiều quốc gia khác nhau nơi mà tiếng Anh được sử dụng như một ngoại ngũ̃. Công cụ này có thể tiếp tục được phát triển trong bối cảnh dạy học tại Việt Nam.

Các nghiên cứu khảo sát đánh giá và phát triển năng lực TPACK của cả sinh viên sư phạm tiếng Anh và giáo viên tiếng Anh với kinh nghiệm giảng dạy khác nhau cho thấy các điểm chung sau đây: (1) Nhận thức của sinh viên sư phạm và giáo viên tiếng Anh về TPACK dao động từ trung bình đến khá cao (2) Mối liên hệ giữa kiến thức TPACK và các thông số như giới tính, tuổi tác và kinh nghiệm dạy học chưa được xác định rõ ràng vì các nghiên cứu cho các kết quả khác biệt nhau. Điều này có thể là do bối cảnh dạy học hay đặc điểm văn hóa xã hội khác nhau.

Kiến thức ứng dụng CNTT (TPACK) được hiểu là năng lực tổng hợp kiến thức sư phạm với kiến thức công nghệ thông tin đặc thù của môn học và kiến thức chuyên môn nhằm xúc tiến quá trình học tập môn học một cách hiệu quả. Mô hình năng lực TPACK này đã thu hút sự quan tâm của nhiều nhà nghiên cứu trên thế giới. Nghiên cứu năng lực TPACK của giáo viên ngoại ngữ là một trong những vấn đề cần thiết đối với công tác đào tạo và phát triển chuyên môn về ứng dụng CNTT trong dạy học ngoại ngữ. Sự đổi mới phương pháp dạy học theo hướng ứng dụng CNTT, thích ứng với yêu cầu của xã hội hiện đại, không thể thiếu những nghiên cứu tìm hiểu và phát triển TPACK trong nhiều bối cảnh dạy học khác nhau tại Việt Nam.

Kết quả nghiên cứu trên thế giới đã mang lại những hiểu biết nhất định về khái niệm TPACK cũng như công cụ đánh giá và khảo sát TPACK. Tuy nhiên trong bối cảnh Việt Nam, rất ít nghiên cứu được thực hiện. Vì vậy cần thiết phải có những nghiên cứu góp phần mở rộng hiểu biết về mô hình TPACK về mặt lý luận và thực tiễn trong bối cảnh dạy học tiếng Anh tại Việt Nam. Ba hướng nghiên cứu có thể thực hiện là

Tiếp tục nghiên cứu phát triển công cụ khảo sát TPACK nhằm phục vụ cho công tác đào tạo và phát triển chuyên môn giáo viên ngoại ngữ. Theo đó công cụ khảo sát của Bostancioğlu và Handley (2018) có thể là một lựa chọn phù hợp.

Khảo sát kiến thức TPACK của sinh viên sư phạm và giáo viên tiếng Anh nói riêng và ngoại ngữ nói chung ở các cấp. Tìm hiểu này có thể góp phần nâng cao sự hiểu biết về năng lực ứng dụng CNTT trong dạy học ngoại ngữ, qua đó xác định nhu cầu và biện pháp nâng cao năng lực TPACK.

Đào tạo và tập huấn về ứng dụng CNTT theo hướng TPACK, đồng thời nghiên cứu hiệu quả của nó đối với việc nâng cao chất lượng dạy học ngoại ngữ.

\section{TÀI LIẸU THAM KHẢO}

Abbitt, J. T. (2011). Measuring technological pedagogical content knowledge in preservice teacher education. Journal of Research on Technology in Education, 43(4), 281-300. https://doi.org/10.1080/15391523.2011.10782573

Anderson, L.W., \& Krathwohl, D. (Eds). (2001). A taxonomy for learning, teaching, and assessing: A revision of Bloom's taxonomy of educational objectives. New York: Longman.

Armstrong, P. (2010). Bloom's taxonomy. Truy cập từ https://cft.vanderbilt.edu/guides-subpages/blooms-taxonomy/ 
Baser, D., Kopcha, T. J., \& Yasar Ozden, M. (2016). Developing a technological pedagogical content knowledge (TPACK) assessment for preservice teachers learning to teach English as a foreign language. Computer Assisted Language Learning, 29(4), 749-764. https://doi.org/ 10.1080/09588221.2015.104745.

Bostancıŏlu, A., \& Handley, Z. (2018). Developing and validating a questionnaire for evaluating the EFL 'Total PACKage': Technological pedagogical content knowledge (TPACK) for English as a foreign language (EFL). Computer Assisted Language Learning, 31(5-6), 572-598. https://doi.org/10.1080/09588221.2017.1422524.

Chai, C. S., Chin, C. K., Koh, J. H. L., \& Tan, C. L. (2013). Exploring Singaporean Chinese language teachers' technological pedagogical content knowledge and its relationship to the teachers' pedagogical beliefs. Asia-Pacific Education Researcher, 22(4), 657-666. https://doi.org/10.1007/s40299-013-0071-3.

Chapelle, C. A (2003). English language learning and technology: Lectures on applied linguistics in the age of information and communication technology. John Benjamins Publishing Company, Amsterdam/Philadelphia.

Davies, G. (2012, February, 2). Computer assisted language learning: Where are we now and where are we going?

http://www.camsoftpartners.co.uk/docs/Futurela b_CALL_Article.htm

Department of Education and Training, Australia Government (n.d). Fact sheet: Competencybased training. https://www.myskills.gov.au/media/1776/backto-basics-competency-based-training.pdf

Harris, J. B., \& Hofer, M. J. (2011). Technological pedagogical content knowledge (TPACK) in action. Journal of Research on Technology in Education, 43(3), 211-229. https://doi.org/ 10.1080/15391523.2011.10782570.

Harris, J., Phillips, M., Koehler, M., \& Rosenberg, J. (2017). TPCK/TPACK research and development: Past, present, and future directions. Australasian Journal of Educational Technology, 33(3), i-viii. https://doi.org/10.14742/ajet.3907

Hoàng Hòa Bình (2015). Năng lực và đánh giá theo năng lực. Tạp chí khoa họ- ĐHSP TP. Hồ Chí Minh, 6(71): 21-32.

Hsu, L. (2016). Examining EFL teachers' technological pedagogical content knowledge and the adoption of mobile-assisted language learning: a partial least square approach. Computer Assisted Language Learning, 29(8), 1287-1297. https://doi.org/10.1080/09588221.2016.1278024.
Kurt, G., Mishra, P., \& Kocoglu, Z. (2013).

Technological pedagogical content knowledge development of Turkish preservice teachers of English. In R. McBride \& M. Searson (Eds.), Proceedings of SITE 2013-Society for Information Technology \& Teacher Education International Conference (pp. 5073-5077). New Orleans, Louisiana, United States: Association for the Advancement of Computing in Education (AACE). Retrieved March 11, 2021 from https://www.learntechlib.org/primary/p/48937/.

Le, N., \& Song, J. (2018). TPACK in a CALL course and its effect on Vietnamese pre-service EFL teachers. Asian EFL Journal, 9(1), 31-56.

Levy, M., (1997). CALL: Context and Conceptualisation. Oxford: Oxford University Press.

Long, M. H. (1996). The role of the linguistic environment in second language acquisition. In W. C. Ritchie, \& T. K. Bhatia (Eds.), Handbook of second language acquisition (pp. 413-468). New York: Academic Press.

Mishra, P., \& Koehler, M. J. (2006). Technological pedagogical content knowledge: A new framework for teacher knowledge. Teachers College Record 108(6), 1017-1054.

Nazari, N., Nafissi, Z., Estaji, M., \& Marandi, S. S. (2019). Evaluating novice and experienced EFL teachers' perceived TPACK for their professional development. Cogent Education, $6(1), 1-26$.

OECD (2018). The OECD Pisa global framework. https://www.oecd.org/education/Globalcompetency-for-an-inclusive-world.pdf

Öz, H. (2015). Assessing EFL pre-service teachers' technological pedagogical content knowledge. International Education Studies, 8(5), 119-131.

Parr, G., Bellis, N. \& Bulfin, S. (2013). Teaching English teachers for the future: Speaking back to TPACK. English in Australia, 48(1), 9-22.

Prasojo, L. D., Habibi, A., Mukminin, A., \& Yaakob, M. F. M. (2020). Domains of technological pedagogical and content knowledge: Factor analysis of Indonesian inservice EFL teachers. International Journal of Instruction, 13(4), 593-608. https://doi.org/10.29333/iji.2020.13437a

Rosenberg, J. M. \& Koehler, M. J. (2015). Context and technological pedagogical content knowledge (TPACK): A systematic review. Journal of Research on Technology in Education, 47(3), 186-210. https://doi.org/10.1080/15391523.2015.1052663.

Saltan, F. \& Arslan, K. (2017). A comparison of preservice and in-service teachers' technological pedagogical content knowledge self-confidence. Cogent Education, 4 (1311501), 1-12. https://doi.org/10.1080/2331186X.2017.1311501 
Sarıçoban, A., Tosuncuoğlu, İ., \& Kırmızı, Ö (2019). A technological pedagogical content knowledge (TPACK) assessment of pre-service EFL teachers learning to teach English as a foreign language. Journal of Language and Linguistic Studies, 15(3), 1122-1138.

Schmid, M., Brianza, E., Petko, D. (2020). Developing a short assessment instrument for technological pedagogical content knowledge (tpack.xs) and comparing the factor structure of an integrative and a transformative model. Computers \& Education, 157(2020), 103967. https://doi.org/10.1016/j.compedu.2020.103967

Schmidt, D. A., Baran, E., Thompson, A. D., Mishra, P., Koehler, M. J., \& Shin, T. S. (2009). Technological pedagogical content knowledge (TPACK): The development and validation of an assessment instrument for preservice teachers. Journal of Research on Technology in Education, 42(2), 123-149.

Shih, C. L., \& Chuang, H. (2013). The development and validation of an instrument for assessing college students' perceptions of faculty knowledge in technology-supported class environments. Computers \& Education, 63, 109-118. https://doi.org/10.1016/j.compedu.2012.11.021
Shulman, L. S. (1987). Knowledge and teaching: Foundations of the new reform. Harvard Educational Review, 57(1), 1-22.

Tai, S.-J. D. (2015). From TPACK-in-action workshops to classrooms: CALL competency developed and integrated. Language Learning \& Technology, 19(1), 139-164. Retrieved from http://lt.msu.edu/issues/february2015/tai.pdf

Tseng, J. (2016). Developing an instrument for assessing technological pedagogical content knowledge as perceived by EFL students. Computer Assisted Language Learning, 29(2), 302-315. https://doi.org/10.1080/09588221.2014.941369.

UNESCO (2015). UNESCO competency framework. Truy cập từ https://en.unesco.org/sites/default/files/competen cy_framework_e.pdf

Voogt, J., \& McKenney, S. (2017). TPACK in teacher education: are we preparing teachers to use technology for early literacy? Technology, Pedagogy and Education, 26(1), 69-83. https://doi.org/10.1080/1475939X.2016.1174730

Willermark, S. (2018). Technological pedagogical and content knowledge: A review of empirical studies published from 2011 to 2016. Journal of Educational Computing Research, 56(3), 315343. https://doi.org/10.1177/07356331177114. 